Please do not remove this page

RMIT

UNIVERSITY

\title{
Geometrogenesis under quantum graphity: Problems with the ripening universe
}

Wilkinson, Samuel; Greentree, Andrew

https://researchrepository.rmit.edu.au/esploro/outputs/9921862752601341/filesAndLinks?institution=61RMIT_INST\&index=null

Wilkinson, S., \& Greentree, A. (2015). Geometrogenesis under quantum graphity: Problems with the ripening universe. Physical Review D (Particles, Fields, Gravitation and Cosmology), 92(8), 1-7.

https://doi.org/10.1103/PhysRevD.92.084007

Document Version: Published Version

Published Version: https://doi.org/10.1103/PhysRevD.92.084007

Repository homepage: https://researchrepository.rmit.edu.au

(C) 2015 American Physical Society

Downloaded On 2023/04/26 20:49:12 +1000

Please do not remove this page 
Thank you for downloading this document from the RMIT Research Repository.

The RMIT Research Repository is an open access database showcasing the research outputs of RMIT University researchers.

RMIT Research Repository: http://researchbank.rmit.edu.au/

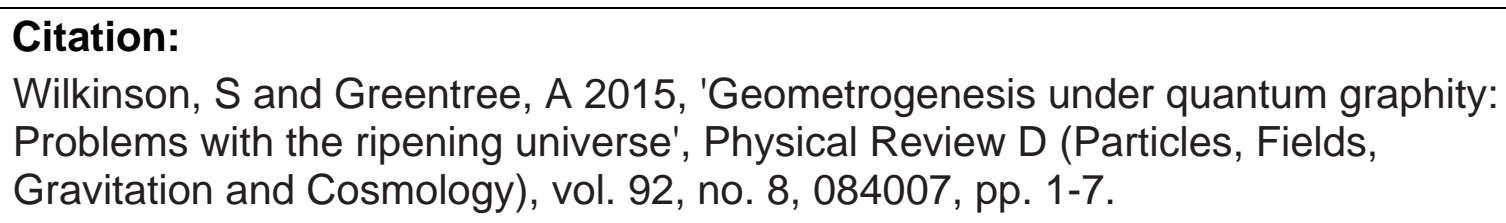

See this record in the RMIT Research Repository at:

https://researchbank.rmit.edu.au/view/rmit:34598

Version: Published Version

\section{Copyright Statement:}

(C) 2015 American Physical Society

Link to Published Version:

http://dx.doi.org/10.1103/PhysRevD.92.084007 
PHYSICAL REVIEW D 92, 084007 (2015)

\title{
Geometrogenesis under quantum graphity: Problems with the ripening universe
}

\author{
Samuel A. Wilkinson ${ }^{1, *}$ and Andrew D. Greentree ${ }^{1,2}$ \\ ${ }^{1}$ Chemical and Quantum Physics, School of Applied Sciences, RMIT University, \\ Melbourne 3001, Australia \\ ${ }^{2}$ Australian Research Council Centre of Excellence in Nanoscale BioPhotonics, RMIT University, \\ Melbourne 3001, Australia \\ (Received 26 June 2015; revised manuscript received 10 September 2015; published 2 October 2015)
}

\begin{abstract}
Quantum graphity (QG) is a model of emergent geometry in which space is represented by a dynamical graph. The graph evolves under the action of a Hamiltonian from a high-energy pregeometric state to a lowenergy state in which geometry emerges as a coarse-grained effective property of space. Here we show the results of numerical modeling of the evolution of the QG Hamiltonian, a process we term "ripening" by analogy with crystallographic growth. We find that the model as originally presented favors a graph composed of small disjoint subgraphs. Such a disconnected space is a poor representation of our Universe. A new term is introduced to the original QG Hamiltonian, which we call the hypervalence term. It is shown that the inclusion of a hypervalence term causes a connected latticelike graph to be favored over small isolated subgraphs.
\end{abstract}

DOI: 10.1103/PhysRevD.92.084007

PACS numbers: 04.60.Pp, 04.60.-m

\section{INTRODUCTION}

The idea that space-time geometry may not be a fundamental feature of the Universe has arisen in several speculative models for the microscopic structure of space [1], in particular those which aim to formulate a background independent theory of quantum gravity [2]. This approach has been taken in research paradigms such as causal dynamical triangulation [3], group field theory [4] and structurally dynamic cellular networks [5]. It has been suggested that the AdS/CFT correspondence in string theory also indicates that space-time is not fundamental [6]. To consider geometry to be merely an effective, coarsegrained property of space-time may resolve some of the conflicts between quantum theory and general relativity [7]. However, this comes at great technical and conceptual cost. A quantum theory with no inherent notion of geometry cannot be formulated with respect to a fixed manifold, nor can it make use of the usual global symmetries. For this reason, models of emergent geometry often use the tools of combinatorics.

One such model is quantum graphity (QG) [8], which postulates that space is represented as a dynamical graph, where points in space are represented by vertices of the graph and adjacencies in space are represented by edges in the graph. The graph is a purely combinatorial object, so there is no a priori notion of geometry. Rather, the model is postulated to possess two distinct thermodynamic phases: a high-temperature pregeometric phase and a low-temperature phase with emergent geometry. The transition between these two phases is called geometrogenesis. QG is a member of

samuel.wilkinson@rmit.edu.au the newly developing family of condensed matter analogue models of spacetime, which treat space or spacetime itself as though it were a many-body system [9].

Here we study the growth (ripening) of grains of the geometric phase starting from the empty graph under the epitaxial approximation (discussed below). We have assumed that the formation of spatial grains from a pregeometric graph follows a process analogous to the growth of crystals. Instead of a homogeneous transition across the entire graph, we argue that as the graph lowers its energy it will tend to form distinct grains which then knit together to form a geometric space. Over time the size of these grains may increase until they are sufficient to house the Universe. This picture for the evolution of the graph lends itself to the formation of domain boundary defects, which have been shown to have observable consequences [10].

Numerical simulations of ripening under the epitaxial approximation reveal a tendency for the model to form a graph consisting of small isolated subgraphs in the lowenergy regime. Here we show that such disjoint graphs are inevitably lower in energy than connected graphs, given the Hamiltonian and parameters first proposed for this model [8]. The formation of a disjoint low-energy graph was first noted in [11], and here we demonstrate that it remains even under more accurate calculations. A disjoint ground state is clearly undesirable for a model that aims to reproduce an extended geometric space. Therefore, we propose a modification to the model which may lead to the formation of a connected space. Under the modified model, introduced in Sec. IV, the honeycomb graph does become a potential ground state; however the epitaxial approach fails to reach this state. 
The low-temperature phase of QG is assumed to be a lattice graph, with the geometry of the lattice corresponding to the emergent geometry of space-time [8]. In particular, parameters of the model are often chosen so that a regular two-dimensional honeycomb graph is favored as the ground state. This is clearly not a representation of our three-dimensional spatial geometry, but is a useful simplification for studying some general features of the model. The high-energy pregeometric state has usually been considered to be the complete graph, in which every vertex is connected to every other. This graph has no geometrical interpretation, making it a candidate for pregeometry. Furthermore, thermodynamic arguments support the notion that higher-temperature graphs have more edges, making the graph with the most edges possible a natural maximum temperature limit [12]. However, the empty graph, in which there exist vertices but no edges, so that every point in space is completely isolated, is also a candidate high-temperature pregeometric graph [11]. This is perhaps more in line with the concept of a Universe arising spontaneously from "nothing," and offers a stronger concept of "spacelessness."

The geometrogenic phase transition has previously been studied in the context of QG $[12,13]$ and in graph models more broadly $[14,15]$; however a finite-temperature phase transition has never been explicitly shown to exist. Since its description in QG, the event of geometrogenesis has been shown to have a concrete realization in causal dynamical triangulations, and may also be present in loop quantum cosmology [16].

\section{MODEL}

QG begins by postulating that space may be represented by a simple, undirected graph $\mathcal{G}$, which is defined by two sets $\mathbb{V}$ and $\mathbb{E}$. $\mathbb{V}$ is the set of vertices (or nodes) on the graph, and $\mathbb{E}$ is the set of edges, which are unordered pairs of the elements of $\mathbb{V}$. Two vertices in $\mathbb{V}, \nu_{i}$ and $\nu_{j}$, are said to be connected (or adjacent) if there exists in $\mathbb{E}$ an edge $\left(\nu_{i}, \nu_{j}\right)$. This graph is dynamic, in the sense that the set of edges may change in time; however the set of vertices is taken to be fixed.

The energetics of the graph are determined by the Hamiltonian,

$$
H=H_{V}+H_{L}+H_{\text {hop }} .
$$

The valence term $H_{V}$ assigns an energy to the graph based on the valence, or degree, of each vertex,

$$
H_{V}=g_{V} \sum_{i} e^{p\left(v_{i}-v_{0}\right)^{2}}
$$

where $v_{i}$ is the valence of the $i$ th vertex, $g_{V}$ is a positive coupling constant, $p$ is a dimensionless real number and $v_{0}$ is the "ideal" valence of the graph. $H_{V}$ is minimized when every vertex in $\mathcal{G}$ has $v_{i}=v_{0}$.
The loops term $H_{L}$ reduces the energy of the graph when there are more loops,

$$
H_{L}=-g_{L} \sum_{L=3}^{L_{\max }} \frac{r^{L}}{L !} \sum_{i} P\left(L, \nu_{i}\right)
$$

where $P\left(\nu_{i}, L\right)$ is a function that counts the number of loops of length $L$ that pass through vertex $\nu_{i}, r$ is a dimensionless real number and $g_{L}$ is a positive coupling constant. For a discussion of the origin of these factors, we refer the reader to [8]. As $r$ is varied, we find that 5-loops are dominant over 6-loops for values of $r$ less than 6, and that 7-loops are dominant over 6-loops for values of $r$ greater than 7 . Between these points, $L^{*}=6$, so that 6-loops contribute most significantly to $H_{L}$. Therefore, we have chosen a value for $r$ in the middle of this region, $r=6.5$. This differs from the value of $r=7.1$ which was used in previous literature [8]. Repeating our calculations with each of these two values of $r$ shows no significant difference to our results except where stated.

Rather than count all loops in the graph, we only consider shortest-path (SP) loops, as these are the most relevant for characterizing a latticelike graph with emergent geometry. SP loops are those that contain no "shortcuts," so that the distance between two vertices along the loop is equal to their distance on the graph. While there exist explicit algebraic formulas for counting the number of loops on a graph up to loops of length 7, there exist no such formulas for SP loops. SP loops are therefore calculated algorithmically using the method presented in [17]. In Ref. [11], loops were counted using explicit algebraic formulas for all loops (not only SP loops) for lengths up to $L=7$. Here we wish to examine graph structures more accurately, so the algorithmic approach of Ref. [17] is always used.

Finally, $H_{\text {hop }}$ is a kinetic term that allows edges to propagate through the graph, changing the configuration of the graph. For a discussion of this term, we again refer the reader to previous literature $[8,11]$.

As discussed in the Introduction, we take the empty graph as the initial state of the system.

\section{RIPENING UNDER THE EPITAXIAL APPROXIMATION}

Calculating the energy for every possible graph is not feasible due to the sheer number of graphs on even a modest number of vertices. Therefore, we make use of the epitaxial approximation as introduced in [11], with the key difference here being our constant use of algorithmic loopcounting methods. In [11], it was shown that when the model evolves epitaxially on 24 vertices from an empty initial graph, it achieves a local minimum 3-regular state which consists of four disjoint subgraphs, and a lowerenergy 4-regular graph which consists of only 3 disjoint subgraphs. This indicates that the graph evolves from the empty starting point by forming small grains and merging 
them together, suggesting a process similar to the ripening that can be seen in many familiar material systems.

In a material system with two phases, one phase may grow to dominance via the process of Ostwald ripening [18]. This process occurs due to atoms being less tightly bound at surfaces than in the bulk of a material, allowing them to detach from small grains and reattach to larger grains. Graphs in QG, however, are nonoriented, so the usual intuition of a "surface" does not exist. However, one may define a surface in $\mathrm{QG}$ as a region of vertices which deviate from the ideal valence (or, away from the ground state, have a larger than average value of $\left|v_{i}-v_{0}\right|$ ). If one accepts this definition, then the difference in binding energy between vertices in the bulk and vertices at the surface is negligible. To be clear, neglecting the loops term in the Hamiltonian, the energy required to reduce the valence of a vertex in the bulk to zero is

$$
\frac{\Delta E_{B}}{g_{V}}=e^{p v_{0}^{2}}-e^{p\left(v_{i}-v_{0}\right)^{2}}
$$

where $v_{i}$ is the valence of the vertices within the bulk. The energy required to reduce the valence of a vertex in the surface to zero is

$$
\frac{\Delta E_{S}}{g_{V}}=e^{p v_{0}^{2}}-e^{p\left(\left|v_{i}-v_{0}\right|+1\right)^{2}} .
$$

On the other hand, the energy required to promote a vertex from the bulk to the surface of a grain is

$$
\frac{\Delta E_{B \rightarrow S}}{g_{V}}=e^{p\left(\left|v_{i}-v_{0}\right|+1\right)^{2}}-e^{p\left(v_{i}-v_{0}\right)^{2}} .
$$

To make this concrete, we calculate these quantities using the parameters of Konopka et al. [8] and taking the valence of the bulk vertices to be 2 . This gives $\Delta E_{B}=4.9017 \times 10^{4}$, $\Delta E_{S}=4.8899 \times 10^{4}$ and $\Delta E_{B \rightarrow S}=118.1903$. Here it can be seen explicitly that the energy difference between the bulk and the surface is negligible compared with the energy required to reduce the valence of either of them to zero.

The model will grow in a way that reduces the number of vertices which have the maximum value of $\left|v_{i}-v_{0}\right|$, which means that when starting from the empty graph the first stage of evolution is to distribute disjoint edges throughout the graph. In the next stage of evolution some of these disjoint edges connect to form small "grains." Eventually we have a graph which consists of many small disjoint subgraphs. It is from this stage that the process we call ripening can begin.

Consider the case of a $k$-regular graph consisting of several disjoint subgraphs where $k<v_{0}$. There are two possibilities for adding a single additional edge: the new edge will either connect two vertices already within the same grain, or it can connect two formerly disjoint grains to each other. Which one is favored generally depends on the definition of loops we employ in $H_{L}$. Loop-counting based exclusively on shortest-path loops favors an edge which connects two formerly disjoint grains, as an internal edge within a grain will destroy some loops already in place. More general loop counting, in which all closed paths contribute to $H_{L}$ (as was used in [11]), favors internal edge formation as this creates more loops, whereas connecting two separate grains does not (any walk that begins in one grain, crosses to the other and then crosses back again to the initial grain must traverse the newly formed edge twice, so such a walk cannot be a path and therefore cannot contribute even in this more general loop counting definition).

The situation from the complete graph is more difficult to interpret, as it is not easy to see which loops exist on the edges when looking only at the holes. In principle, evolution from the complete case should be able to give rise to grains of geometric space, as well as phase separation where some regions are in the pregeometric state while others exhibit local emergent geometry. However, when the graph is already connected the different grains will be difficult to identify. Furthermore, algorithmic loop counting is computationally prohibitive when dealing with highly connected graphs.

The evolution from the complete graph to a low-energy $v_{0}$-regular graph under the epitaxial approximation was simulated for $N=24$ and $N=36$ using parameters from Konopka et al. [8] $\left(g_{V}=1, g_{L}=1 / 500, v_{0}=3\right.$ and $p=1.2$ ), with $r=6.5$ as discussed above. Important steps in the evolution of the $N=36$ are presented in Fig. 1 .

The general features of the evolution presented in Fig. 1 are mostly the same as those that were seen in the $N=24$ case. The only significant difference is the fact that the $v_{0}$ regular state on $N=24$ consists entirely of the symmetrical cubic structures seen in Fig. 1(d), whereas it is not possible to populate a graph on 36 vertices entirely with cubes as 36

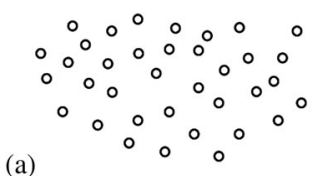

(a)

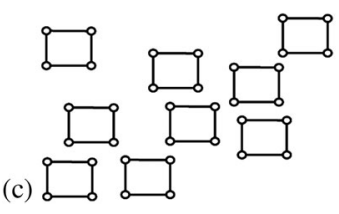

(b)

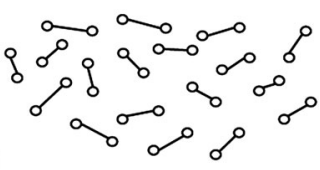

(d)

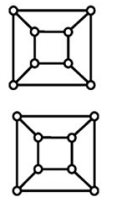

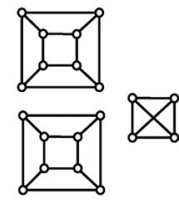

FIG. 1. (a) The epitaxial growth of spatial domains starting with the empty graph as the initial graph. (b) The graph always evolves in a way that reduces the number of vertices with the maximum value of $\left|v_{i}-v_{0}\right|$, so the first step of evolution is the formation of isolated edges. (c) Isolated edges then form 4-loops, which knit together (d) in a process we call "ripening" to form cubic structures. The graph in (d) is a local minimum, and is lower in energy than a connected, isotropic honeycomb lattice for the values of the parameters we have used. 
is not divisible by 8 , so a small $K_{4}$ forms from the "leftover" vertices. Thus we have a frustrated ground state. Frustration is expected to be negligible in the $N \rightarrow \infty$ limit.

Somewhat surprisingly, the formation of isolated 4-loops in step (c) of Fig. 1 is more favorable than the formation of isolated 6-loops. This is because, although 6-loops reduce the energy more, the graph is able to produce more 4-loops, so the overall effect is a lower energy. Furthermore, epitaxial growth will favor the formation of 4-loops as these minimize the instantaneous energy at each step in evolution. Even more surprisingly, the ground state configuration in Fig. 1(d) consists of isolated cubes, and not a connected honeycomb lattice. This cubic state is lower in energy than a connected, isotropic honeycomb lattice for the same values of the model parameters, the honeycomb having an energy of 29.4048 while the isolated cubes have an energy of 29.0779. This difference is small, but it is enough to demonstrate that the honeycomb graph is not the ground state, and the energy gap is expected to grow with $N$ (see Fig. 3 and the surrounding discussion). The honeycomb graph does contain more 6-loops than the isolated cubes [the honeycomb has $2 / 3$ loops per vertex, while the isolated cubes have $1 / 2$ in the ideal case and $2 / 9$ with the $K_{4}$ defect seen in Fig. 1(d)]; however the cubes also have many 4-loops. It is possible to increase the value of the Hamiltonian parameter $r$ to a value such that the 4-loops become negligible. For example, the honeycomb is lower in energy than the isolated cubes for $r=7.1$ (the value used in previous work on QG [8]); however at this value 7-loops dominate over 6-loops. If a connected regular graph were to be favored at all with this value, it would be a graph of heptagons, which would constitute a hyperbolic rather than a flat geometry. The crucial consequence of this discussion is that the connected honeycomb graph cannot be the ground state of the model, and this generic effect of favoring disconnected over connected graphs is likely to hold for other parameter choices.

The process of ripening happens primarily between steps (c) and (d) in Fig. 1. Two "squares" in (c) knit together to form "cubes." The cubes form one by one under the epitaxial approximation. The formation of a single cube from two squares is shown in Fig. 2.

In more traditional descriptions of ripening in material systems, one of the key quantities of interest is the average radius of particles as a function of time (as well as other factors such as the coefficient of diffusion). For abstract graphs with no inherent notion of geometry, the radius of the graph is defined as the smallest eccentricity of the graph, the diameter the largest eccentricity, where the eccentricity of a vertex is the largest possible geodesic distance to any other vertex on the graph. The case of ripening in QG as shown in Fig. 2 is interesting because the diameter of the grains does not increase monotonically. Rather, we see the diameter of the grains undergo a sharp increase from 2 to 5 between steps (a) and (b), followed by a decrease to 3 in step (c) (where it remains). This "two steps forward, one step back" type of growth is also seen

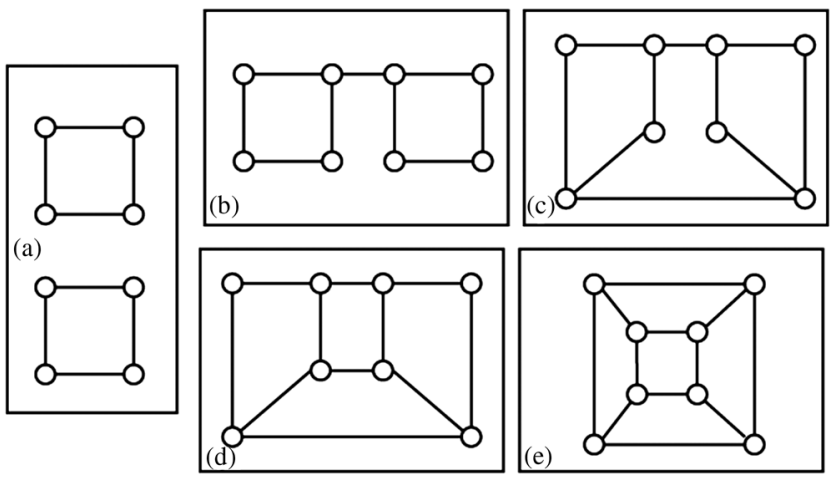

FIG. 2. The evolution of the graph from one consisting of 4-cycles seen in Fig. 1(c) to one constituted by cubic structures seen in Fig. 1(d) is presented step by step. Each subfigure in sequence represents the formation of one additional edge. Interestingly, the diameter of each grain increases from 2 in (a) to 4 in (b), before decreasing back to 3 in (c). This is in contrast to the material version of Ostwald ripening, where the average diameter of large particles grows monotonically.

when the graph transitions from one made up of isolated edges to one consisting of isolated 4-loops. The radius of the grains, however, does increase monotonically from 2 to 3 . If the graph theoretic concepts of radius and diameter apply to the emergent geometry, this implies a picture where space forms by expansion, followed by slight contraction. However, the extent to which the graph theoretic notion of diameter of the grains corresponds to the geometric concept of the diameter of a space is unclear.

Another way in which the evolution observed here differs from ripening in material systems is that the process occurs mostly homogeneously. The 4-cycles in Fig. 1(c) form one at a time, but cubic structures do not begin to form until there are no remaining vertices of degree 1. Likewise, the 4-cycles themselves do not begin to form until there are no remaining vertices of degree 0 . The graph goes through distinct stages of evolution, but goes through them uniformly. This is in contrast to the nucleation and phase separation that is observed when material systems undergo ripening. This feature is most likely a consequence of both the epitaxial approximation and the form of $H_{V}$, which causes evolution to focus on vertices of the maximum value of $\left|v_{i}-v_{0}\right|$ above all else.

Our simulation shows that the formation of two separate grains in this model is preferable to a regular lattice spreading out from the boundary of a honeycomb grain. We may then expect that a QG model with the empty graph as a high-energy starting point tends to form disjoint subgraphs. Such disconnected states can be lower in energy than a connected, extended lattice. Indeed, the cubic graph seen in Fig. 1(d) is lower in energy than an isotropic connected honeycomb lattice on the same number of vertices. This undermines an assumption of the model that the Hamiltonian in Eq. (1) favors an extended honeycomb lattice. 


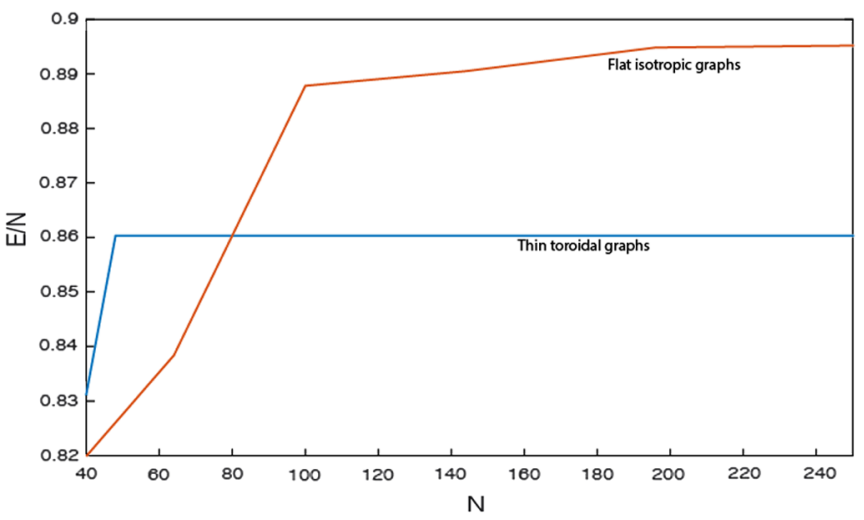

FIG. 3 (color online). Plot of the energy per vertex of various graphs as a function of the number of vertices $N$, using parameters based on Konopka et al. [8]. The red line shows the energy per vertex of isomorphic toroidal honeycomb graphs, where the major and minor circumferences are equal. The blue line shows honeycomb graphs where the minor circumference is fixed at 6 , and only the major circumference is varied. For comparison, with our parameters a graph made up of isolated cubes always has an energy per vertex of 0.6385 , which is notably lower than any of these honeycomb graphs. From this figure, the $N \rightarrow \infty$ limit is not certain, but there does not seem to be any indication that larger subgraphs will be more favorable at large $N$.

Honeycomb lattice grains have the topology of a flat torus, so that there are no "loose ends" (no vertices with $v_{i} \neq v_{0}$ ). This can be thought of as a rectangle with opposite edges identified, tiled with a honeycomb lattice. The "lengths" of the two sides of this rectangle (alternatively, the minor and major circumference of the torus) are important, as when the sides are of length 6 then 6-loops can be formed by circumnavigating the space, not just by the plaquettes in the lattice. This increases the number of 6-loops and thereby decreases the energy. So, for a grain of a given $N$ which we assume to be connected, the lowestenergy honeycomb configuration will be a torus which is long and thin, so that 6-loops can wrap around the width of the torus.

In Fig. 3, we show the energy of various graphs as a function of $N$, as calculated with algorithmic loop counting. The blue curve shows graphs which are flat tori with a minor circumference of 6 , so that 6-loops may form by winding around the width of the torus. Therefore $N$ is changed by changing the major circumference, i.e. making the torus "longer." The red curve shows the energy per vertex of isotropic toroidal graphs, where the major and minor circumferences are equal. It can be see that the 6-by6 torus does indeed have the lowest energy per vertex of the honeycomb graphs shown, with the energy per vertex of the long thin tori becoming constant for large $N$. A graph made up of isolated cubes has an energy per vertex of 0.6385 with our parameters, which is significantly lower than any of the honeycomb graphs presented in Fig 3. This shows a clear tendency for the formation of a disconnected space, which would make the model a poor description of our Universe. This is discussed further in the Conclusion.

It is worth noting that, although it is not always possible to tile an arbitrary space with a honeycomb lattice (see, for example, a buckyball, which must include some 5-loops in order to adopt spherical topology), this is a geometric constraint and has no bearing on the topology that a graph may adopt.

\section{HYPERVALENCE}

The existence of a connected latticelike ground state is an important requirement of QG. All of the terms in the Hamiltonian and all of the values used in our calculations were originally tailored to favor a connected honeycomb graph as the low-energy state; however as we have seen there exist disjoint states of lower energy. The simplest solution to this problem is to modify the QG Hamiltonian in Eq. (1) by introducing a term which will drive connectivity. In particular, here we introduce a generalization of the valence term $H_{V}$ [Eq. (2)], which we call the "hypervalence."

While the valence depends on the degree of each vertex, the hypervalence also depends on the 2-degree and 3 -degree, i.e. the number of vertices at distance 2 and 3 from the initial vertex. The hypervalence energy is defined as

$$
\begin{aligned}
H_{H V} \equiv & \sum_{i} g_{v 1} e^{p_{1}\left(v_{i, 1}-v_{1}\right)^{2}}+g_{v 2} e^{p_{2}\left(v_{i, 2}-v_{2}\right)^{2}} \\
& +g_{v 3} e^{p_{3}\left(v_{i, 3}-v_{3}\right)^{2}}
\end{aligned}
$$

and the parameters here are all analogous to those in the valence term. $v_{i, d}$ is the number of vertices at distance $d$ from vertex $i, g_{v d}$ is a positive real number determining the strength of the $d$ th term and $p_{d}$ is a dimensionless real number determining the penalty for deviation from the ideal $d$-degree, $v_{d}$.

In principle, the number of terms within the hypervalence term could extend to $N$. Equation (7) has been restricted to three terms for computational tractability, and therefore the weighting parameters $g_{v d}$ and $p_{d}$ must be selected so as to be negligible for $d>3$. Rapidly decaying couplings also imply a kind of pseudolocality in the model-vertices an arbitrary distance away do affect the energy contribution from a given vertex, but this contribution quickly becomes negligible as the distance increases.

For the modified Hamiltonian to give rise to a regular ground state, we require $v_{d}=d v_{1}$. In keeping with the original model, we set $v_{1}=v_{0}=3, g_{V 1}=g_{V}=1$ and $p_{1}=p=1.2$. For higher-degree terms to contribute negligibly, coupling constants were chose to scale as $g_{V d}=$ $\left(g_{V 1} / d !\right)^{d}$ and the $p_{d}$ were fixed so that the argument of the exponential of each term in the hypervalence is equal for the empty graph. This leads to $p_{3}=4 p_{2} / 9=p_{1} / 4$. Other values are in principle possible, so long as higher-degree terms contribute significantly less than lower-degree terms. 

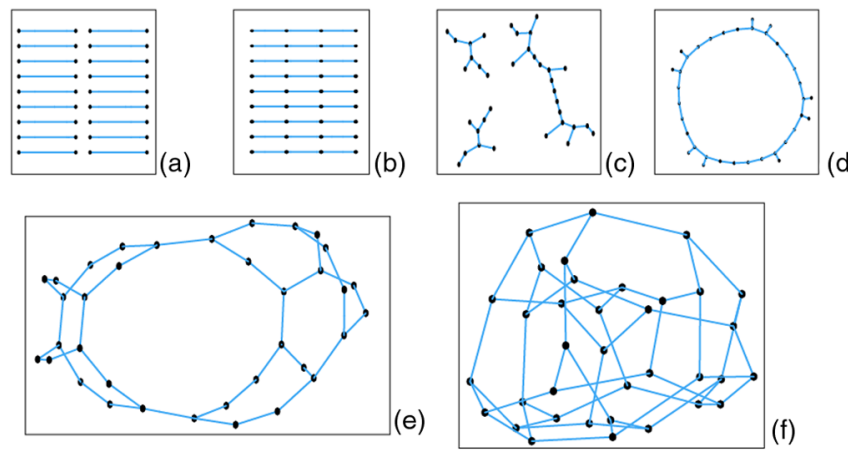

FIG. 4 (color online). Stages of the ripening process under the hypervalence term. Graphs are shown after the formation of (a) 18, (b) 27, (c) 33, (d) 36, (e) 43 and (f) 54 edges. It can be seen that this does favour a connected graph, but the low energy state in (f) is not latticelike and there is no immediately evident emergent geometry. The lowest energy state here is still higher in energy than the regular two-dimensional honeycomb lattice, indicating a failure of the epitaxial approximation to arrive at the ground state of the model.

Numerical simulation of the ripening of this new model was performed starting from an empty graph on $N=36$ under the epitaxial approximation, with various stages of the ripening process along with the final low-energy state shown in Fig. 4. This low-energy graph is connected, making it a better representation of a continuous space than the low-energy graph in Fig. 2. However it is still not the kind of latticelike graph hoped for by the QG program, nor does it have any obvious interpretation in terms of an emergent geometry.

Explicit calculations show, however, that with the hypervalence term, an isotropic connected honeycomb graph is lower in energy than both the isolated cubes and the low-energy state found by the epitaxial approximation shown in Fig. 4. The honeycomb graph has an energy of 38.5950, while the graph in Fig. 4(f) has an energy of 46.2645 and the isolated cubes shown in Fig. 1(d) have an energy of $5.0830 \times 10^{4}$. There are two important implications of this: (1) the addition of the hypervalence term is sufficient to favor an extended, flat, latticelike ground state, and (2) the epitaxial approximation is not sufficient to find the ground state. The insufficiency of the simple epitaxial approximation employed here implies the importance of higher-order process, where multiple edges may be added or deleted simultaneously. However, simulating such processes is computationally difficult and beyond the scope of this work.

\section{CONCLUSION}

Ripening under the epitaxial approximation leads to the formation of a disconnected space. Clearly, a disconnected space is not a good representation of the Universe in which we live. The concept of disconnected space may hint at some interpretation in terms of a multiverse scheme, but only if any of the individual grains in the graph are large enough to support the large extended Universe we find ourselves in. This tendency to form disconnected spaces was noticed previously [11], and was a key motivation for this work.

The epitaxial approximation considered in this work only allowed for one-edge processes, where only one edge may be created or deleted at any time. Extending this approach to include the possibility of two-edge processes, where either two edges are created or deleted simultaneously, or where two edges hop to different positions simultaneously, may radically alter the graph dynamics presented here. It must be assumed that such processes are lower-probability events, but if they are sufficiently energetically favorable when compared with single-edge processes they may still dominate, or at least influence, graph evolution.

We have demonstrated that the honeycomb graph is not the ground state of the original QG model. Since the model was designed to give the honeycomb graph as a ground state, this may be considered a partial failure of the model. However, we have demonstrated that a simple extension of the model to include a hypervalence term restores the possibility that the connected honeycomb graph may be the ground state. Furthermore, we have shown that the epitaxial approximation employed in [11] is not sufficient to return the true ground state of the model, at least when the model is extended to include a hypervalence term.

We have proposed a solution to this connectivity problem by including an additional term in the Hamiltonian, which we have called "hypervalence." This term depends not only on the degree of each vertex but also on the 2- and 3-degree (and, ideally, all higher degrees in a rapidly converging manner). Hypervalence is a natural extension of the valence term in the original model, and its inclusion leads the model to favor a connected graph. While isolated cubes were found to have lower energy than a connected honeycomb lattice in the original model, the honeycomb lattice does become a lower-energy state and a potential ground state when the hypervalence term is considered. However, the lowest-energy state found under an epitaxial approach was not the honeycomb graph, nor any other latticelike graph with a clear interpretation as a geometrical space. The lowest-energy state attained by this approach was also higher in energy than the honeycomb graph, which indicates a failure of the epitaxial approximation to reproduce the QG ground state.

\section{ACKNOWLEDGMENTS}

A. D. G. acknowledges the Australian Research Council for financial support (Contract No. DP130104381). We would like to thank Bill Moran for helpful comments and conversations. 
[1] L. Sindoni, Symmetry Integrability Geom. Methods Appl. 8, 027 (2012).

[2] F. Markopoulou, New directions in background independent quantum gravity, in Approaches to Quantum Gravity, edited by D. Oriti (Cambridge University Press, Cambridge, England, 2009), p. 129.

[3] J. Ambjørn, J. Jurkiewicz, and R. Loll, Phys. Rev. Lett. 93, 131301 (2004).

[4] D. Oriti, Proc. Sci., QG-PH (2007) 030 [arXiv:0710.3276].

[5] M. Requardt and S. Rastgoo, arXiv:1501.00391.

[6] N. Seiberg, arXiv:hep-th/0601234.

[7] D. Oriti, Stud. Hist. Phil. Mod. Phys. 46, 186 (2014).

[8] T. Konopka, F. Markopoulou, and S. Severini, Phys. Rev. D 77, 104029 (2008).

[9] J. Bain, Stud. Hist. Phil. Mod. Phys. 44, 338 (2013).
[10] J. Q. Quach, C.-H. Su, A. M. Martin, and A. D. Greentree, Phys. Rev. D 86, 044001 (2012).

[11] S. A. Wilkinson and A. D. Greentree, Phys. Rev. D 90, 124003 (2014).

[12] F. Caravelli and F. Markopoulou, Phys. Rev. D 84, 024002 (2011).

[13] T. Konopka, Phys. Rev. D 78, 044032 (2008).

[14] S. Chen and S. S. Plotkin, Phys. Rev. D 87, 084011 (2013).

[15] F. Conrady, J. Stat. Phys. 142, 898 (2011).

[16] J. Mielczarek, arXiv:1404.0228.

[17] D. S. Franzblau, Phys. Rev. B 44, 4925 (1991).

[18] L. Ratke and P. W. Voorhees, Growth and Coarsening: Ostwald Ripening in Materials Processing (SpringerVerlag, Berlin, 2002). 